\section{References}

Di H, Cameron K, Shen J, Winefield C, O’Callaghan M, Bowatte $\mathrm{S}$ et al. (2010). Ammonia-oxidizing bacteria and archaea grow under contrasting soil nitrogen conditions. FEMS Microbiol Ecol 72: 386-394.

Di HJ, Cameron KC, Shen JP, Winefield CS, O'Callaghan $\mathrm{M}$, Bowatte $\mathrm{S}$ et al. (2009). Nitrification driven by bacteria and not rchaea in nitrogen-rich grassland soils. Nat Geosci 2: 621-624.

Jia Z, Conrad R. (2009). Bacteria rather than archaea dominate microbial ammonia oxidation in an agricultural soil. Environ Microbiol 11: 1658-1671.

Leininger S, Urich T, Schloter M, Schwark L, Qi J, Nicol GW et al. (2006). Archaea predominate among ammonia-oxidizing prokaryotes in soils. Nature 442: 806-809.

Martens-Habbena W, Berube PM, Urakawa H, de la Torre JR, Stahl DA. (2009). Ammonia oxidation kinetics determine niche separation of nitrifying archaea and bacteria. Nature 461: 976-979.

Moissl C, Bruckner JC, Venkateswaran K. (2008). Archaeal diversity analysis of spacecraft assembly clean rooms. ISME J 2: 115-119.

Offre P, Prosser JI, Nicol G. (2009). Growth ofammoniaoxidizing archaea in soil microcosms is inhibited by acetylene. FEMS Microbiol Ecol 70: 99-108.

Schauss K, Focks A, Leininger S, Kotzerke A, Heuer H, Thiele-Bruhn S et al. (2009). Dynamics and functional relevance of ammonia-oxidizing archaea in two agricultural soils. Environ Microbiol 11: 446-456.

Tourna M, Freitag TE, Nicol GW, Prosser JI. (2008). Growth, activity and temperature responses of ammonia-oxidizing archaea and bacteria in soil microcosms. Environ Microbiol 10: 1357-1364.

Wuchter C, Abbas B, Coolen MJ, Herfort L, van Bleijswijk J, Timmers $\mathrm{P}$ et al. (2006). Archaeal nitrification in the ocean. Proc Natl Acad Sci USA 103: 12317-12322.

\title{
Creating and characterizing communities of human gut microbes in gnotobiotic mice
}

Jeremiah J Faith*, Federico E Rey*, David O’Donnell, Maria Karlsson, Nathan P McNulty, George Kallstrom, Andrew L Goodman and Jeffrey I Gordon

The ISME Journal (2010) 4, 1094-1098; doi:10.1038/ ismej.2010.110; published online 22 July 2010

Microbiology laboratories are laden with flasks, plates, and freezer stocks containing axenic cultures and their products. In contrast, virtually every other habitat on Earth is filled with microbial communities of varying degrees of complexity. In this context, microorganisms are interdependent components of ecosystems; deciphering this dynamic requires a move from microbial organisms studied in isolation to model microbial communities studied under conditions that mimic those encountered by their members in their native habitats. Here, we focus on model communities consisting of microbes that inhabit the human body habitat containing our largest collection of organisms-the gut.

The adult human gastrointestinal tract is a microbial bioreactor, containing all three domains of life. This ecosystem is teeming with microorganisms at its distal end $\left(10^{11}-10^{12}\right.$ cells $\mathrm{ml}^{-1}$ luminal contents in the colon) and less so at its proximal end (an estimated $10^{3}$ $10^{4}$ cells ml ${ }^{-1}$ luminal contents in the duodenum). The gut microbiota affects myriad aspects of our systems physiology, ranging from processing and harvesting of macronutrients and micronutrients (and xenobiotics!) from our diets, to shaping the features of our innate and adaptive immune system. Recently, deep sampling of the fecal microbial community has revealed that each of us harbor a collection of a several hundred bacterial phylotypes (Qin et al., 2010; Turnbaugh et al., 2010). The exact set of microbes differs from person to person although there is a greater degree of similarity between family members (Turnbaugh et al., 2009a, 2010). A catalog of several million genes present in the fecal microbiome has been assembled from analysis of a 577Gbp data set obtained from shotgun sequencing of fecal community DNA prepared from 124 Europeans (Qin et al., 2010) and a 10.1-Gbp data set generated from a set of deeply sampled obese monozygotic co-twins living in the United States (Turnbaugh et al., 2010). These data sets provide a starting point for making in silico predictions about functions that can be attributed to the gut microbiota. Measurements of expressed mRNAs (Turnbaugh et al., 2010), proteins (Verberkmoes et al., 2009), and metabolites (Hoverstad et al., 1984; Li et al., 2008; Martin et al., 2008) in gut samples represent a first step toward testing these predictions.

\section{Generating germ-free mice via embryo transfer}

Germ-free (GF) mice provide a complementary approach for characterizing the properties of the human gut microbiome. Methods for establishing and 
propagating inbred strains of mice under GF conditions were established $>50$ years ago by several groups. Re-derivation was based on caesarian section of a conventionally raised (microbe laden) mother, passaging the intact uterine horns containing the pups through a germicidal bath, and delivery of her pups in a GF isolator where they were suckled by a lactating foster mother (the original GF foster mothers were generated by caesarian delivery of litters, hand feeding of pups in an GF isolator with an autoclaved artificial liquid diet until a male and female reached reproductive maturity; colonies were established from these GF progenitors and their offspring distributed to other gnotobiotic facilities; for example see http://gordonlab.wustl.edu/SuppInfo/Reyniers Sacksteder_1957.pdf). This approach requires precise timing and is also inefficient: in our experience, 0-5 wild-type pups survive to weaning age per re-derivation $(n=100 \mathrm{C}$-sections performed between 1998 and 2007). Therefore, we have replaced this method with embryo transfer: embryos are harvested 1 day after mating, and transferred under sterile conditions to a pseudopregnant GF mother generated by mating to a vasectomized GF male (Table 1). This technique yields 5-8 live born animals/25 embryos transferred/recipient mother ( $n>250$ procedures). GF status is verified by PCR of feces using universal bacterial 16S rRNA gene primers and by culturing fecal and skin swabs under conditions that support growth of a broad range of anaerobic and aerobic bacterial species and fungi.

\section{Studies of complex microbial communities in gnotobiotic mice}

GF mice can be colonized with microbial communities of varying complexity and origin at defined stages of their life. For example, gut microbial communities can be harvested from various body habitats of conventionally raised mice with defined genotypes and physiological phenotypes, and introduced into GF recipients (possessing a desired genotype) to determine how much of the donor phenotype is transferable to the resulting conventionalized mice via the microbiota. If complete or even partial phenotypic transfer occurs, follow-up studies can be performed to define composition of the donor community, the mechanisms by which the donor community impacts host physiology, and how the recipient affects the transplanted microbiota/ microbiome. These types of studies have typically been performed using gut contents (Turnbaugh et al., 2006; Vijay-Kumar et al., 2010), but in principle can be extended to communities harvested from any body habitat.

We have developed procedures for subjecting GF and conventionalized mice to a variety of surgical and non-surgical manipulations while maintaining their gnotobiotic environments: these procedures include (1) endurance training through swimming in a warmed sterile water tank placed within the isolator (a 'gnotatorium'; Crawford et al., 2009); (2) using a plexiglass gnotobiotic transporter to bring mice to an irradiator for whole body irradiation; (3) bone marrow transplantation after whole body irradiation using marrow harvested from animals of varying genotypes (Crawford and Gordon, 2005); (4) using specialized transporters that fit inside a magnetic resonance imager to determine adiposity; and (5) techniques for generating aggregation chimeras using morula-stage embryos (for a discussion and illustration of why the stem cell hierarchy of intestinal crypts make chimeric mice such a powerful tool for studying cell autonomous versus non-autonomous regulation in the gut epithelium, see Wong et al., 2000).

We have also validated procedures for transplanting human fecal microbial communities into GF mouse

Table 1 Procedure for generating GF mice via embryo transfer

Step 1: A 6-10-week-old female GF Swiss-Webster mouse is checked for estrus and mated to a vasectomized GF Swiss Webster male; the female is checked for a vaginal plug the next day; if plugged, the mouse is transferred to a $2 \mathrm{X} 2 \mathrm{X} 2$ foot flexible film gnotobiotic isolator containing a sterilized transfer pod, a sterilized anesthetic solution, and sterilized surgical equipment (all sterilizations are performed in the port of the gnotobiotic isolator by fogging the materials with a solution of chlorine dioxide (Clidox-S)).

Step 2: Embryos are harvested from 3-4-week-old conventionally raised, super-ovulated females of the desired genotype 1 day after they have been mated to conventionally raised males of the desired genotype. Using a laminar flow tissue culture hood, embryos are obtained by flushing the fallopian tubes with Brinster's BMOC-3 medium supplemented with penicillin (10000 units ml $\left.{ }^{-1}\right)$ and streptomycin $\left(10 \mathrm{mg} \mathrm{ml}^{-1}\right)$. They are then cleaned by serial washes in drops of the same medium (6-10 wash cycles of $2-4$ min each; note that these drops are submerged in embryo-tested sterile mineral oil (Sigma)).

Step 3: The pseudopregnant GF Swiss-Webster female from step 1 is anesthetized, placed in the transfer pod, brought into a laminar flow hood, and an incision made along the back to expose ovary and oviduct. The oviduct is then pierced with a 22-gauge needle. The needle is withdrawn and embryos are transferred (25/recipient mother) in BMOC-3/antibiotic solution into the oviduct using a microtransfer pipette, analogous to the ones used for pronuclear injections of oocytes when generating transgenic animals.

Step 4: The ovary and oviduct are placed back into the abdomen, the dorsal incision is closed, the anesthetized animal is placed in the transfer pod, and the pod is put into the port of the gnotobiotic isolator. The pod is then chemically sterilized with chlorine dioxide for 15-20 min, re-introduced into the gnotobiotic isolator, and the mother is allowed to revive (a warm-heating pad is placed below the plastic isolator). One to two pseudopregnant mice can be processed at the same time using this procedure. 
recipients that are then fed diets that do or do not resemble those of the human donors (Turnbaugh et al., $2009 \mathrm{~b})$. We have found that a remarkable proportion of human fecal microbial diversity can be transferred in this manner even if the donor specimen had been frozen at $-80^{\circ} \mathrm{C}$ for $1-2$ years (all bacterial phyla, up to $90 \%$ of class-level and genus-level taxa, and $60-90 \%$ of species level-phylotypes in donor samples are identifiable in recipient mice using $16 \mathrm{~S}$ rRNA-based pyrosequencing). Once engrafted, the transplanted human microbial communities are remarkably stable, can be reliably transmitted across generations of animals, and exhibit well defined and reproducible biogeographical features along the length of the mouse gut (Turnbaugh et al., 2009b). Efficient intergenerational transfer of transplanted human fecal microbiota allows the microbiota and the host's innate/adaptive immune system to co-evolve beginning at birth in 'second generation' mice. 'Humanized' gnotobiotic mice can be used for proof-of-mechanism studies that cannot be readily conducted in humans where potentially confounding variables, including variations in host genotype, diet, and antibiotic consumption are notoriously difficult to control. A derivative of this procedure is to capture as much diversity as possible by culturing a donor's fecal microbiota, and then transferring this culture collection en masse to wild-type or genetically engineered GF recipients (culturable 'humanized' mice).

\section{Assembling defined model communities in vivo using gnotobiotic mice}

As more members of the human gut microbiota are cultured and their genomes sequenced (Nelson et al., 2010), an opportunity exists to create model human gut communities in gnotobiotic mice where all community members and their complement of microbial genes are known. Members present in these synthetic human gut microbial communities can be selected from culture collections based on various criteria, including their consistent association with specific human physiologic or pathophysiologic states, their representation in a fecal microbiota that when transferred en masse confers a phenotype to recipient GF mice, their phylogenetic features, and/or by the results of in silico predictions of their functions based on inspection of their genomes. These communities can be used to address a number of basic questions in the field: for example (1) to what extent do priority effects, where established species are able to sequester limiting space or resources and are thereby able to exclude potential colonizers, determine community composition; (2) what is the strength of interspecific interactions (a key to generating predictive models of community structure and dynamics; Trosvik et al., 2010); (3) what are the genetic predictors of niche overlap; (4) how robust are the assembled communities to various environmental perturba- tions; and (5) what is the microbial host range of viruses and the determinants of viral lifecycles in various regions of the gut ecosystem (Reyes et al., 2010).

To date, these model communities have consisted of as few as 2 and as many as 15 members and have been used to explore some of the metabolic interactions that take place in the distal gut (both microbial-microbial and microbial-host; see Sonnenburg et al., 2006; Denou et al., 2009; Mahowald et al., 2009; Rey et al., 2010). These communities have also been extremely useful for technology development. For example, if the complete genome sequence of each member is known, then the relative abundance of each member can be used to infer the proportional representation of genes encoding various functions (for example metabolic and signaling pathways) in that community using quantitative metagenomic methods (Morgan et al., 2010). With the current capacity of the Illumina GAIIx DNA sequencer $(\sim 30$ million reads per lane), relative and absolute species abundance is quantifiable for all microbes representing at least $0.01 \%$ of the community, while allowing $\geqslant 100$ barcoded samples to be pooled in a single lane of the eight-lane flow cell for multiplex sequencing. These inexpensive assays of community member abundance support the large sample sizes needed for computational modeling of the responses of a defined (synthetic) community to various perturbations (including systematic alterations in macronutrient and micronutrient composition of the diet), across time.

Understanding how different gut communities modulate their gene expression in response to changes in diet, host physiological status, or invasion with microbial species is another key step in understanding the operations of the gut microbiota. RNA-Seq allows quantification of transcriptomes at high resolution and over a broad dynamic range. In the case of synthetic communities, where all the species and genes are known, this high-resolution data can be used to verify gene structure/operons, generate in silico reconstructions of expressed metabolic pathways for each member in the community, and make predictions concerning the metabolic niches of each species. These predictions can be informed by RNA-Seq analysis of individual community members during monoculture under highly defined conditions (for example minimal medium supplemented with systematically varied carbon sources), then be validated using quantitative mass spectrometry-based analyses of products of microbial metabolism. These studies can prompt follow-up, hypothesis-based studies of metabolic niches where the investigator manipulates the species used to construct these model communities, or uses other approaches to perturb the activities of key members in ways that provide proof-of-principle tests for affecting community function and host physiology (for example devising ways to manipulate the hydrogen economy of the gut to affect the 
efficiency of fermentation and host energy extraction; Rey et al., 2010). In addition, gnotobiotic mice harboring defined collections of sequenced organisms provide an opportunity to further develop methods for extracting and characterizing, by LC-MS, the proteins expressed by their model microbiota (peptides can be readily mapped as all genes are known; Mahowald et al., 2009).

Community genetics provides another powerful technology to dissect the operations of microbial communities and to identify potential avenues (targets) for microbiome-directed therapeutics. Addition or removal of organisms before gavaging the model microbiota into GF mice provides the simplest genetic perturbation to identify species that confer a benefit or detriment to other community members or the host. Another method is insertion sequencing, which combines genome-wide transposon mutagenesis with massively parallel sequencing (Goodman et al., 2009). In this approach, complex populations of tens of thousands of transposon mutants of a given sequenced community member are generated and simultaneously introduced into wild-type or genetically manipulated GF mice in the presence or absence of other (sequenced) microbes. The representation of each mutant in the input community is determined by targeted, sequencing of transposon-adjacent chromosomal DNA, and compared with their representation in the output community recovered from the mouse. Differences in mutant representation in input versus output communities indicate which microbial genes confer a fitness advantage as a function of whatever selective pressure is intentionally applied to the system (Goodman et al., 2009).

\section{Creating more realistic defined microbial communities: the challenges ahead}

A look to the near future reveals a number of pressing needs. With genome sequences available for almost 200 human gut isolates from eight bacterial phyla and Archaea, our ability to move toward larger model communities in gnotobiotic mice is limited by our ability to grow microbes in parallel; therefore, we need to identify media capable of supporting growth of diverse sets of microbes, scale-up methods for growing anaerobic cultures in parallel (for example move from tubes to 96-well plates or microfluidic chips with individually addressable strains), and modify sequencing pipelines to allow for rapid assays of purity of single cell-derived cultures. The current set of sequenced human gut bacteria isolates are largely from different individuals. Using microbial communities obtained from a single individual is desirable for reasons described above, including the fact that co-existing microbial species have co-evolved, creating distinct collections of strain-level phylotypes. Thus, to move toward increasingly realistic communities, we need high-throughput methods to isolate and array in multi-well plates, single cell-derived cultures of hundreds of bacteria from a single individual. Sequencing capacity will likely be available to many individual laboratories in the next few years to generate draft genomes from hundreds of these arrayed organisms. In the context of human microbiome projects, the ultimate informative model microbiota would contain microbes isolated from single individuals that confer the donor's phenotype to the recipient gnotobiotic mice. The full model community 'tool kit,' both experimental and computational (including application of existing and new methods for modeling) could be applied to these communities in an attempt to expedite understanding of how their component organisms and genes confer a donor phenotype. However, for these efforts to benefit and build the field, we also need to create the infrastructure necessary to readily share both communities and their associated data between laboratories. The knowledge base for model microbial community biology (conditions for culturing its members, microbial genome sequences, quantitative data about community membership as a function of various perturbations, associated meta-transcriptome, meta-proteome, and metabolomic data sets; information about their impact on host physiology) requires systems for data deposition, annotation, and retrieval, which combine computer automation and error checking with as little human curation as necessary. Finally, currently license agreements, biological safety regulations, and shipping procedures are designed to distribute individual strains or multiple variants of the same strain. We must streamline the regulatory and infrastructure hurdles for multi-species distribution to ensure that the best model communities developed over the coming years have the opportunity to earn their 'model' designation as they follow the path of Escherichia coli and Bacillus subtilis as facilitators of biological discovery.

\section{Acknowledgements}

We thank the members of our laboratory for their support, and Tore Midtvedt for providing us with the 1957 report of Reyniers and Sacksteder about derivation and breeding of GF mice. Work from the laboratory described in this report was supported by grants from the NIH (DK30292, DK70977, DK78669), the Crohn's and Colitis Foundation of America, the Bill and Melinda Gates Foundation, and Groupe Danone.

JJ Faith, FE Rey, D O’Donnell, M Karlsson, NP McNulty, G Kallstrom, AL Goodman and JI Gordon are at Center for Genome Sciences and Systems Biology, Washington University School of Medicine, St Louis, MO, USA. E-mail: jgordon@wustl.edu *These two authors contributed equally to this work. 


\section{References}

Crawford PA, Crowley JR, Sambandam N, Muegge BD, Costello EK, Hamady $\mathrm{M}$ et al. (2009). Regulation of myocardial ketone body metabolism by the gut microbiota during nutrient deprivation. Proc Natl Acad Sci USA 106: 11276-11281.

Crawford PA, Gordon JI. (2005). Microbial regulation of intestinal radiosensitivity. Proc Natl Acad Sci USA 102: 13254-13259.

Denou E, Rezzonico E, Panoff JM, Arigoni F, Brussow H. (2009). A mesocosm of Lactobacillus johnsonii, Bifidobacterium longum, and Escherichia coli in the mouse gut. DNA Cell Biol 28: 413-422.

Goodman AL, McNulty NP, Zhao Y, Leip D, Mitra RD, Lozupone CA et al. (2009). Identifying genetic determinants needed to establish a human gut symbiont in its habitat. Cell Host Microbe 6: 279-289.

Hoverstad T, Fausa O, Bjorneklett A, Bohmer T. (1984). Short-chain fatty acids in the normal human feces. Scand J Gastroenterol 19: 375-381.

Li M, Wang B, Zhang M, Rantalainen M, Wang S, Zhou H et al. (2008). Symbiotic gut microbes modulate human metabolic phenotypes. Proc Natl Acad Sci USA 105: 2117-2122.

Mahowald MA, Rey FE, Seedorf H, Turnbaugh PJ, Fulton RS, Wollam A et al. (2009). Characterizing a model human gut microbiota composed of members of its two dominant bacterial phyla. Proc Natl Acad Sci USA 106: 5859-5864.

Martin FP, Wang Y, Sprenger N, Yap IK, Lundstedt T, Lek P et al. (2008). Probiotic modulation of symbiotic gut microbial-host metabolic interactions in a humanized microbiome mouse model. Mol Syst Biol 4: 157.

Morgan JL, Darling AE, Eisen JA. (2010). Metagenomic sequencing of an in vitro-simulated microbial community. PLoS One 5: e10209.

Nelson KE, Weinstock GM, Highlander SK, Worley KC, Creasy HH, Wortman JR et al. (2010). A catalog of reference genomes from the human microbiome. Science 328: 994-999.

Qin J, Li R, Raes J, Arumugam M, Burgdorf KS, Manichanh C et al. (2010). A human gut microbial gene catalogue established by metagenomic sequencing. Nature 464: 59-65.
Rey FE, Faith JJ, Bain J, Muehlbauer MJ, Stevens RD, Newgard CB et al. (2010). Dissecting the in vivo metabolic potential of two human gut acetogens. J Biol Chem 285: 22082-22090.

Reyes A, Haynes M, Hanson N, Angly FE, Heath AC, Rohwer F et al. (2010). Viruses in the fecal microbiota of monozygotic twins and their mothers. Nature 466: 334-338.

Sonnenburg JL, Chen CT, Gordon JI. (2006). Genomic and metabolic studies of the impact of probiotics on a model gut symbiont and host. PLoS Biol 4: e413.

Trosvik P, Rudi K, Straetkvern KO, Jakobsen KS, Naes T, Stenseth NC. (2010). Web of ecological interactions in an experimental gut microbiota. Environ Microbiol. E-pub ahead of print. doi: 10.1111/j.1462-2920.2010.02236.x

Turnbaugh PJ, Hamady M, Yatsunenko T, Cantarel BL, Duncan A, Ley RE et al. (2009a). A core gut microbiome in obese and lean twins. Nature 457: 480-484.

Turnbaugh PJ, Ley RE, Mahowald MA, Magrini V, Mardis ER, Gordon JI. (2006). An obesity-associated gut microbiome with increased capacity for energy harvest. Nature 444: 1027-1031.

Turnbaugh PJ, Quince C, Faith JJ, McHardy AC, Yatsunenko T, Niazi $\mathrm{F}$ et al. (2010). Organismal, genetic, and transcriptional variation in the deeply sequenced gut microbiomes of identical twins. Proc Natl Acad Sci USA 107: 7503-7508.

Turnbaugh PJ, Ridaura VK, Faith JJ, Rey FE, Knight R, Gordon JI. (2009b). The effect of diet on the human gut microbiome: a metagenomic analysis in humanized gnotobiotic mice. Sci Transl Med 1: 6ra14.

Verberkmoes NC, Russell AL, Shah M, Godzik A, Rosenquist M, Halfvarson J et al. (2009). Shotgun metaproteomics of the human distal gut microbiota. ISME J 3: 179-189.

Vijay-Kumar M, Aitken JD, Carvalho FA, Cullender TC, Mwangi S, Srinivasan S et al. (2010). Metabolic syndrome and altered gut microbiota in mice lacking Toll-like receptor 5. Science 328: 228-231

Wong $\mathrm{MH}$, Saam JR, Stappenbeck TS, Rexer $\mathrm{CH}$, Gordon JI. (2000). Genetic mosaic analysis based on Cre recombinase and navigated laser capture microdissection. Proc Natl Acad Sci USA 97: 12601-12606. 